\title{
Corrigendum
}

\section{Hirotsu $\mathrm{N}$ and Wright $\mathrm{M}$ (2002). Using a Markov process model of an association football match to determine the optimal timing of substitution and tactical decisions}

Journal of the Operational Research Society (2002) 53, 1174. doi:10.1057/palgrave.jors.2601414

This paper contains the following errors, for which we apologise.

1. Equations (8) and (9) should be replaced by

$$
\begin{aligned}
& \Omega_{\mathrm{m}}(T+\mathrm{d} t) \\
& =\max \begin{cases}\mathrm{A}_{\mathrm{m}} \Omega_{\mathrm{m}}(T) \mathrm{d} t+\Omega_{\mathrm{m}}(T) & \text { no substitution should } \\
& \text { yet be made with } \\
& \text { time } T \text { remaining } \\
\mathrm{A}_{\mathrm{m}} \Omega_{\mathrm{s}}(T) \mathrm{d} t+\Omega_{\mathrm{s}}(T) & \text { now making the } \\
& \text { substitution }(M \rightarrow S) \\
& \text { at time } T \text { remaining }\end{cases}
\end{aligned}
$$

$$
\begin{aligned}
& \Omega_{\mathrm{s}}(T+\mathrm{d} t) \\
& =\max \begin{cases}\mathrm{A}_{\mathrm{s}} \Omega_{\mathrm{s}}(T) \mathrm{d} t+\Omega_{\mathrm{s}}(T) & \begin{array}{l}
\text { not changing tactics } \\
(\text { tactics } \mathrm{S} \rightarrow \mathrm{S})
\end{array} \\
\mathrm{A}_{\mathrm{s}} \Omega_{\mathrm{m}}(T) \mathrm{d} t+\Omega_{\mathrm{m}}(T) & \begin{array}{l}
\text { changing tactics } \\
(\text { tactics } \mathrm{S} \rightarrow \mathrm{M})
\end{array}\end{cases}
\end{aligned}
$$

3. Equations (12) and (13) should be replaced by

$$
\Omega_{\mathrm{m}}(T+\mathrm{d} t)
$$$$
=\max \begin{cases}\mathrm{A}_{\mathrm{m}} \Omega_{\mathrm{m}}(T) \mathrm{d} t+\Omega_{\mathrm{m}}(T) & \text { not changing tactics } \\ \int_{T-\Delta T_{1}}^{T} \mathbf{A}_{\mathrm{m}} \Omega_{\mathrm{m}}(t) \mathrm{d} t & (\mathrm{M} \rightarrow \mathrm{M}) \\ +\int_{T-\Delta T_{1}-\Delta T_{2}}^{T-\Delta T_{\mathrm{s}}} \mathbf{A}_{\mathrm{s}}(t) \mathrm{d} t & (\mathrm{M} \rightarrow \mathrm{S}) \\ +\Omega_{\mathrm{s}}\left(T-\Delta T_{1}-\Delta T_{2}\right) & \end{cases}
$$

$$
\begin{array}{ll}
\Omega_{\mathrm{s}}(T+\mathrm{d} t) & \\
=\mathrm{A}_{\mathrm{s}} \Omega_{\mathrm{s}}(T) \mathrm{d} t+\Omega_{\mathrm{s}}(T) & \text { a substitution has already } \\
& \text { been made and there } \\
& \text { is time } T \text { remaining }
\end{array}
$$

2. Equations (10) and (11) should be replaced by

$$
\begin{aligned}
& \Omega_{\mathrm{m}}(T+\mathrm{d} t) \\
& =\max \begin{cases}\mathrm{A}_{\mathrm{m}} \Omega_{\mathrm{m}}(T) \mathrm{d} t+\Omega_{\mathrm{m}}(T) & \begin{array}{l}
\text { not changing tactics } \\
\text { (tactics } \mathrm{M} \rightarrow \mathrm{M})
\end{array} \\
\mathrm{A}_{\mathrm{m}} \Omega_{\mathrm{s}}(T) \mathrm{d} t+\Omega_{\mathrm{s}}(T) & \begin{array}{l}
\text { changing tactics } \\
(\text { tactics } \mathrm{M} \rightarrow \mathrm{S})
\end{array}\end{cases}
\end{aligned}
$$

$\Omega_{\mathrm{s}}(T+\mathrm{d} t)$

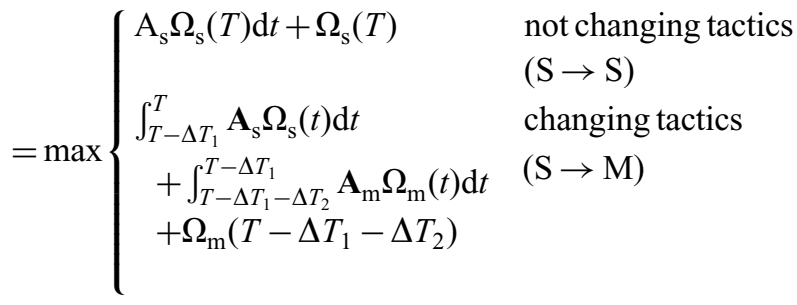

\section{Reference}

1 Hirotsu N and Wright M (2002). Using a Markov process model of an association football match to determine the optimal timing of substitution and tactical decisions. J Opl Res Soc 53: 88-96.

\section{University of Lancaster}

